\title{
Braiding dynamics in semiflexible filament bundles under oscillatory forcing
}

\author{
Valentin M. Slepukhin ${ }^{a}$ and Alex J. Levine $\mathrm{e}^{a, b}$
}

\begin{abstract}
We examine the nonequilibrium production of topological defects - braids - in semiflexible filament bundles under cycles of compression and tension. During these cycles, the period of compression facilitates the thermally activated pair production of braid/anti-braid pairs, which then may separate when the bundle is under tension. As result, appropriately tuned alternating periods of compression and extension should lead to the proliferation of braid defects in a bundle so that linear density of these pairs far exceeds that expected in thermal equilibrium. Secondly, we examine the slow extension of braided bundles under tension, showing that their end-to-end length creeps nonmonotonically under a fixed force due to braid deformation and the motion of the braid pair along the bundle. We conclude with a few speculations regarding experiments on semiflexible filament bundles and their networks.
\end{abstract}

\section{Introduction}

The mechanics of semiflexible filaments has been a subject of broad interest both for its role in the mechanics of the cytoskeleton and as a testing ground for various principles of polymer and soft condensed matter physics. One feature of biological filaments, such as F-actin and collagen (a key constituent of the extracellular matrix) is their ability to form densely cross-linked bundles. These bundles are composed of a number of nearly parallel filaments cross linked by one of a variety of specialized proteins.

Previous research has focused on the collective mechanical response of permanently cross-linked filament bundles $\frac{1}{1}$, showing that the bundle inherits a complex, scale-dependent bending modulus due to cross linking, even though the bending mechanics of the constituent filaments is comparatively simple. In most circumstances of biological interest, cross linkers detach and attach to filament bundles and their networks. As a result, such structures acquire a viscoelastic response - their stress relaxation has a complex time dependence and these systems dissipate work not only through viscous dissipation in the surrounding fluid but also by linker unbinding. As a result, the collective mechanical response of networks of filament bundles have a nontrivial lowfrequency viscoelastic response at frequencies below a characteristic linker unbinding rate 2 .

We explore here particular type of stress relaxation through the production and movement of defects in cross-linked bundles.

\footnotetext{
${ }^{a}$ Department of Physics \& Astronomy, University of California, Los Angeles. 90095 USA.

${ }^{b}$ Department of Chemistry \& Biochemistry, University of California, Los Angeles. 90095 USA.
}

Previously, we have shown that bundles support a set of topological defects - loop, braids, and dislocations ${ }^{4}$. The lifetime of these defects is quite long, growing with the length of the bundle, since they cannot be removed by local rearrangements of the cross linking on the bundle. Defects, however, can be produced in defect/anti-defect pairs by local rearrangements, and defect pair production is predicted to be enhanced by applied compressive loads 5 . In this article, we report on theoretical studies of defect pair production under reciprocal mechanical deformations and consider how the proliferation of defects affects the forceextension relation of a bundle in a pulling-velocity-dependent manner.

The motion of topological defects plays a critical role in the long-time plastic deformation of crystalline solids under mechanical loading. Defect motion in plays a similar role in the slow relaxation of bundles under load. Mechanical loading can also generate defect pair production. We first consider pair production in cycles of compression and extension of one bundle. We then examine the force extension relation of defected bundles by examining the extension of the bundle as a function of time for fixed force.

\section{The piece-wise linear defect potential and the de- fect distribution}

We have shown that the production of braid/anti-braid pairs in a compression bundle can be mapped onto the Kramers escape problem in one dimension using a single reaction coordinate 5 . The two defects must be first produced together in the form of defect/anti-defect pairs, which requires energy $E_{\text {defect }}$. The formation of one defect introduces a length mismatch between the 
A

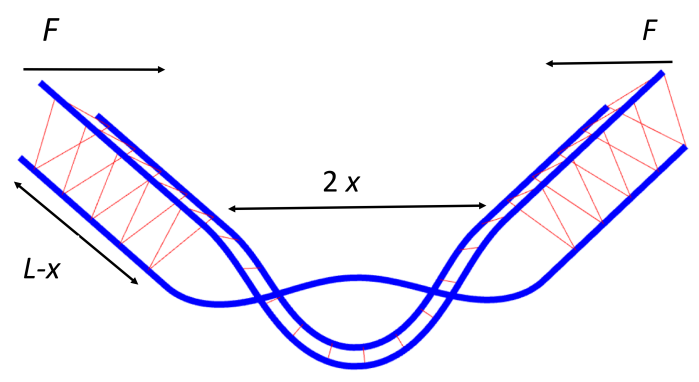

C

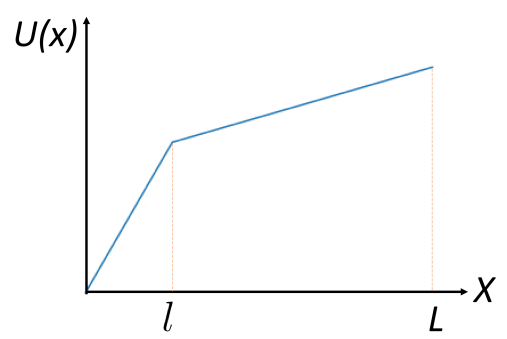

B

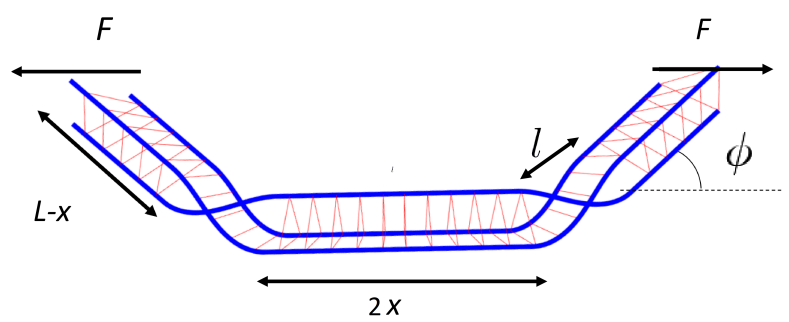

D

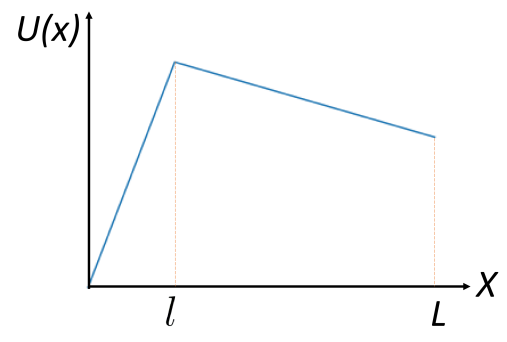

Fig. 1 Schematic illustration: (A) A pair of braids produced under compression force $F$ on a semiflexible filament bundle of length $2 L$ (and comprised of three filaments), but not separated. $2 x$ is the size of the defected region. The filaments are shown in blue while the cross links are shown in red. (B) Pair of braids is separated under tension. The braids produce a kink with angle $\phi .2 x$ is the distance between braids (including their size). In the lower figures we show the piece-wise linear potential $U(x)$ under compression (C) and under extension (D). The left part of the potential $x<l$ corresponds to the production of defects, the while right $x>l$ controls the separation of the defects.

filaments involved that is then compensated by the second, antidefect. As a result, defect pair production entails only local rearrangements of cross linkers. During this point in the thermallyactivated production of the defect pair, we may take the single reaction coordinate to represent the total length "exchanged" between defects. Once the defects separate so that a region of crosslinked bundle appears between them, the defects can no longer exchange length, but they may separate along the bundle by reptative motion. Most importantly, since the defects generate localized bends or kinks under external loading, the motion of the defects changes the end-to-end distance of the bundle under load. By separating the energy of the system decreases. During this separation (under external loading) the distance between the defects plays the role of the reaction coordinate.

Taking these two aspects of the problem together, we may consider the stochastic pair production process as the thermal escape of a single fictitious particle, representing the reaction coordinate $x$, in an approximately piece-wise linear potential. Before defect pair separation $x<l$, where $l$ is the size of the defect at the moment of the separation, the potential increases linearly as more length is exchanged between the defect pair. The exact answer would require taking into account all different pathways from properly cross-linked bundle to the bundle with braids. We make the simplest approximation, i.e., a linear potential, motivated by the fact that we need to remove number of cross links proportional to the size of the uncross-linked region. Then the effective potential is $U(x)=A x$, with $A=E_{\text {defect }} / l$. The energy of the defect incorporates bending energy of the filaments, missing binding en- ergy of the cross links not present in the defected region, and the work of the applied force:

$$
E_{\text {defect }}=E_{\text {bending }}(\phi)+E_{\text {binding }}(\phi)-2 F(L-x) \cos (\phi)-2 F x \text {, }
$$

where $\phi$ is the angle formed by a single braid (see Figure11), $F$ is a force acting on the ends of the bundle (positive sign is chosen for the extension), $2 L$ is a length of the bundle. The angle $\phi$ is determined by energy minimization with respect to it. As defects separate, $x$ grows. The angle also changes, but as soon as $x \ll L$, this change contributes to the energy at the next order in the small parameter $x / L$. We address the explicit dependence of the angle on coordinate in the Section 4 Omitting this effect, we can again write an effective potential $U(x)=A l+B(x-l)$ with $B=$ $2 F(\cos \phi-1)$. Thus, we may explore the pair production process using the potential

$$
U(x)=\left\{\begin{array}{l}
A x, x<l \\
A l+B(x-l), l<x<L \\
\infty, x>L
\end{array}\right.
$$

Before considering dynamics, we use this potential to consider the equilibrium distribution of defects on a bundle of length $L$. Specifically, we consider the equilibrium separation of two defects. Using Eq. 2 it is trivial to write the probability distribution in this potential: $p(x)=\frac{1}{Z} e^{-\beta U(x)}$ where the partition function $Z$ is given by

$$
Z=\int_{0}^{L} e^{-\beta U(x)} d x
$$


and $\beta=1 / k_{\mathrm{B}} T$. A straightforward calculation arrives at the partition function written as the sum of two parts corresponding to the two pieces of the potential

$$
Z=Z_{1}+Z_{2}
$$

with

$$
Z_{1}=\frac{1}{\beta A}\left(1-e^{-\beta A l}\right)
$$

and

$$
Z_{2}=\frac{1}{\beta B} e^{-\beta(A-B) l}\left(e^{-\beta B l}-e^{-\beta B L}\right) .
$$

Taking the ratio of these partition sums we obtain the ratio of observable separated braid pairs to strongly interacting and colocalized braids:

$$
Z_{2} / Z_{1}=\frac{A e^{-\beta(A-B) l}\left(e^{-\beta B l}-e^{-\beta B L}\right)}{B\left(1-e^{-\beta A l}\right)}
$$

From the above we see that in thermal equilibrium we expect there to be a low density of separated braids, at least at low temperatures $(T \approx 300 \mathrm{~K})$. Specially, if the thermal energy is much lower than the cross-linker binding energy (which is typically true in biopolymer filament systems) we expect an exponentially small density of braids $\left(\propto e^{-E_{\text {braid }} \beta}\right.$, where for known filaments $\beta E_{\text {braid }} \gg 1$, the smallest is for DNA with $\beta E_{\text {braid }} \approx 50$. Braid pairs, however, can be generated either during bundle formation or via cycles of compression and expansion, as would be expected in a bundle network under reciprocal shear.

\section{The nonequilibrium braid distribution in a time- dependent potential}

When one applies a time-varying force, the effective potential controlling the production and motion of the braids also changes in time. As a result, we cannot rely on the equilibrium distribution discussed in the previous section. Instead, we have to solve the Smoluchowski diffusion equation for defect density $\rho(t, x)$ :

$$
\partial_{t} \rho(t, x)=D \partial_{x}\left[\left(\partial_{x}-\beta F(t, x)\right) \rho(t, x)\right],
$$

where the force now takes the form

$$
F(t, x)=\left\{\begin{array}{l}
A(t), x<l \\
B(t), l<x<L .
\end{array}\right.
$$

During compression $A(t)=A_{c}$ and $B(t)=B_{c}$. During expansion $A(t)=A_{s}, B(t)=B_{s}$ (note that $B_{s}$ is negative). In the above, $D$ is the defect diffusion constant. We cannot solve this equation analytically, however, we can provide a qualitative analysis. To simplify, we assume that $A$ and $B$ are fixed during each period of compression and expansion. We explore how the defect production rate depends on the lengths of these periods of compression and extension. We also estimate the maximal defect production rate.

The transport time from 0 to $l$ in the potential is controlled by the constant $A$. This is braid pair production rate when the braids have stored length of $l$. This problem is simply first passage time $^{\sqrt{6}}$ to reach $l$ in the linearly increasing potential, which we may estimate to be

$$
T_{0 l}=\frac{1}{D} \frac{e^{\beta A l}}{\beta^{2} A^{2}} .
$$

Similarly, we estimate the transport time from $L$ to $l$. This gives an estimate of the lifetime of the braid pair, since when their separation returns to $l$ they will likely annihilate. Here we must distinguish two limiting cases. In the first case, we consider purely diffusive braid motion and in the second, we look at the deterministic transport of the braids under an applied force using a mobility set by the diffusion constant and the Einstein relation. We find

$$
T_{L l}=\left\{\begin{array}{l}
L^{2} / D, B \beta>1 / L \\
L /(B \beta D), B \beta<1 / L .
\end{array}\right.
$$

In general, where we expect there to be both diffusive and advective defect motion, we find that the time for defects to recombine is

$$
T_{L l}=\frac{L^{2}}{D(1+\beta B L)}
$$

In the limit of large $L, T_{L l} \gg T_{0 l}$ so that the time for distant defect pairs to come together and potentially annihilate is much greater than their production time. If braids are able to separate sufficiently, we expect this ordering of time scales to be valid and thus predict braid proliferation on the bundle.

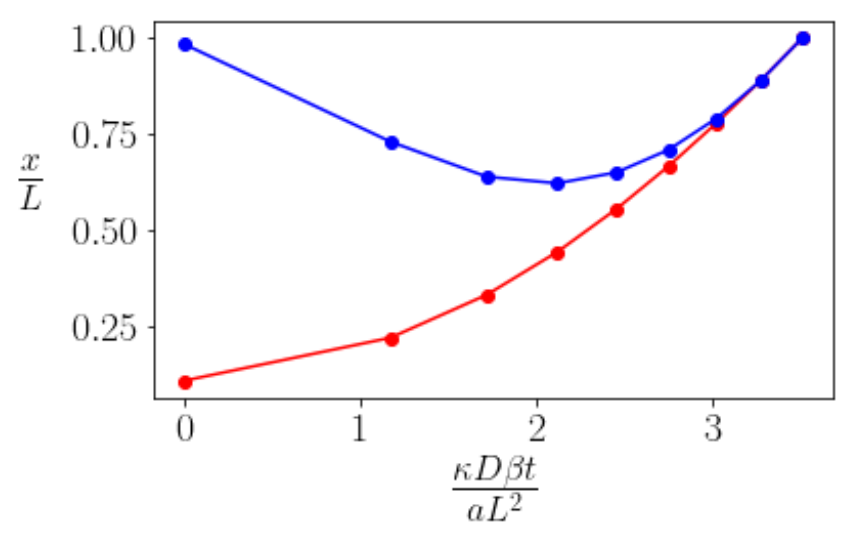

Fig. 2 The time evolution of the distance between the defects (red) and the distance between the ends of the bundle (blue) under a constant applied tensile force $F=0.9 \frac{\kappa}{a L}$ plotted as a function of non-dimensionalized time. The cross linker binding energy is $\mu=4 \frac{\kappa}{a^{2}}$, where $\kappa$ is bending modulus of a single filament, and $a$ is the size of the cross-link plus two radii of the filament (the distance between the center lines of the cross linked filaments).

Because the production time $T_{0 l}$ has an exponential dependence on $A$,

$$
T_{0 l}^{\text {compression }} \ll T_{0 l}^{\text {stretching }}
$$

since the $A$ parameter is much larger under stretching than it is under compression: $A_{c}<A_{s}$. If we choose the time-dependence of the applied force so that the stretching time $\tau_{s}$ and compression $\tau_{c}$ satisfy the inequalities

$$
T_{0 l}^{\text {compression }} \ll \tau_{c} \ll \tau_{s} \ll T_{0 l}^{\text {stretching }} \ll T_{L l},
$$


we may analyse the dynamics of the system using a few approximations.

Since the braid production rate during compression is small compared to the compression time: $T_{0 l}^{\text {compression }} \ll \tau_{c}$, then during the compression period, the density on the left $0<x<l$ equilibrates. Since $\tau_{s} \ll T_{0 l}^{\text {compression }} \ll T_{L l}$, during the stretching period, the probability density $\rho(x)$ decreases near the potential maximum at $x=l$, but it is highly unlikely that thermally excited hopping over the barrier at $l$ occurs. Since $\tau_{c} \ll \tau_{s} \ll T_{0 l}^{\text {compression }} \ll T_{L l}$, the applied force is changing sufficiently fast that the density of the right of of the potential $x>l$ may be replaced by its time-averaged value. Moreover, since $\tau_{c} \ll \tau_{s}$, density on the right of the potential is effectively determined by the dynamics during the stretching period. Because the compression period is shorter, the already produced braids are unlikely to be driven together and annihilate. As a result, we conclude that the density distribution on the left is effectively determined by the compression period and the density on the right by the stretching period. Finally, we note that the Smoluchowski diffusion equation requires the continuity of both the probability density and its current at the boundary $x=l$. From these conditions, we obtain a value of the averaged density on the right as a function of the equilibrium density on the left. This implies that with this sequence of inequalities, the braid production and separation may be considered to take place in a time-averaged, effective potential where the production part is set by the compression forces and braids separate under a force related to extension. Specifically, we consider

$$
U(x)=\left\{\begin{array}{l}
A_{c} x, x<l \\
A_{c} l+B_{s}(x-l), l<x<L \\
\infty, x>L .
\end{array}\right.
$$

In this potential, the probability of braids on the left $x<l$ will be proportional to

$$
Z_{1}=\frac{1}{\beta A_{c}}\left(1-e^{-\beta A_{c} l}\right),
$$

while on the right

$$
Z_{2}=\frac{1}{\beta B_{s}} e^{-\beta\left(A_{c}-B_{s}\right) l}\left(e^{-\beta B_{s} l}-e^{-\beta B_{s} L}\right) .
$$

The ratio is

$$
Z_{2} / Z_{1} \approx-\frac{A_{c}}{B_{s}} e^{-\beta\left(A_{c}-B_{s}\right) l} e^{-\beta B_{s} L} \gg 1
$$

which exceeds the case of only compression by a factor of $e^{\beta\left|B_{s}\right| L}$, and the case of only expansion by a factor of $e^{\beta\left(A_{s}-A_{c}\right) l}$ - see Eq.7.

\section{Constant force stretching dynamics of a braided bundle}

We now consider an experiment in which one stretches a previously compressed bundle (by laser tweezers or other means) at a fixed force and determines the time rate of change of the bundle's length. This is akin to a step force rheological measurement, and is closely related to determining the force-extension curve of a filament or filament bundle. Typically, in such force extension measurements, one considers the limit of slow extension so that the observed length corresponds the thermal equilibrium prediction under a fixed force ${ }^{7}$. In this case, however the extension of the bundle will be time dependent even though the force is constant.

To study this problem, we minimize the energy of the bundle under a fixed stretching force. By doing so, we assume that bending of the kink angles at the braids is fast compared to the time scale of measurement of the end-to-end distance. We do not, however, assume that the advection and diffusion of the braids is similarly fast. Doing this energy minimization numerically, we obtain the time dependence shown in Fig. 2. The time dependence of the bundle's extension is nonmonotonic: as defects diffuse from each other, the bundle initially becomes shorter. This happens due to the fact that as $x$ grows, the $L-x$ becomes smaller, hence, the moment of the force decreases as well. Since the stretching force decreases, kinks become less stretched and their angles increase, decreasing end-to-end distance.

\section{Summary}

Semiflexible filament bundles admit three classes of topological defects: loops, braids, and dislocations. These defects are all likely to be produced in the formation of bundles from solutions of semiflexible filaments by the introduction of cross-linking agents. They are, however, unlikely to form spontaneously in thermal equilibrium when the filament bundles are also chemically equilibrated with a reservoir of cross linkers. Here we have pointed out that reciprocal compression and extension of filament bundles, however, is capable of producing a higher nonequilibrium density of braid defects within a bundle. The key insight is that bundles under compression can locally buckle producing braid, anti-braid pairs. Upon subsequent extension, these braid pairs will be driven to separate, as long as they do not immediately annihilate. In order to assure that braid pairs produced in the previous compression cycle (and separated during the previous extension part of that cycle) do not annihilate during the subsequent compressive cycle, one needs to introduce an asymmetry between the period of compression (short) and the period of extension (long). Other cycles of compression and extension will also produce braid, anti-braid pairs, but at lower density. We predict that the short compression period following long extension one will result in the maximum possible defect density.

We also examined the extensional dynamics under a fixed tensile load of semiflexible bundles containing a braid defect pair. Here one does not observe the standard worm-like chain force extension relation at very low frequencies. The extension of the bundle is not controlled by the depletion of the length reservoir associated with the thermally-generated undulations of the bundle, but rather by the motion of the braid defects and the bending of the kinks associated with these defects. In essence, our predictions refer to the analog of plastic deformation in solids associated with defect motion rather than the (entropic) elastic response of the bundle, which, due to cross linking, is suppressed. We note that the end-to-end distance of the bundle varies nonmonotonically with time under a constant tensile load. This somewhat counter-intuitive result occurs due to the combination of two ef- 
fects: braid separation, which lengthens the bundle, and kink angle relaxation, which shortens it.

For experimental verification of these predictions, there is no more direct measurement than compression/extension experiments on individual semiflexible filament bundles using laser or magnetic tweezers to manipulate the bundle's stress state $\frac{8}{}$. We are currently unaware of such studies, but look forward to seeing them. Although less direct in testing the predictions made here, standard shear measurements and studies of stress relaxation in networks of filament bundles with transient cross linkers at long times or low frequencies are particularly relevant to the present work. We imagine that, at sufficiently long times, stress relaxation will be dominated by plastic deformation of network comprised of both tearing and reattachment of bundles from each other, and the plastic deformation of the individual bundles themselves, presumably following the mechanisms discussed here. We do not, as yet, understand how to distinguish these dynamics in rheological data, and this remains one of the principal open questions related to this work.

\section{Acknowledgements}

The authors acknowledge partial support from grant no. NSFDMR-1709785. V.M.S. also acknowledges support from the Bhaumik Institute Graduate Fellowship, and UCLA Dissertation Year Fellowship.

\section{Author Contributions}

Conceptualization, Alex Levine; Formal analysis, Valentin Slepukhin; Funding acquisition, Alex Levine; Investigation, Valentin Slepukhin; Methodology, Alex Levine; Project administration, Alex Levine; Software, Valentin Slepukhin; Supervision, Alex Levine; Visualization, Valentin Slepukhin; Writing âĂŞ original draft, Valentin Slepukhin and Alex Levine; Writing âĂŞ review \& editing, Valentin Slepukhin and Alex Levine.

\section{Notes and references}

1 C. Heussinger and E. Frey, The European Physical Journal E, 2007, 24, 47-53.

2 C. P. Broedersz, M. Depken, N. Y. Yao, M. R. Pollak, D. A. Weitz and F. C. MacKintosh, Phys. Rev. Lett., 2010, 105, 238101.

3 K. W. Müller, R. F. Bruinsma, O. Lieleg, A. R. Bausch, W. A. Wall and A. J. Levine, Phys. Rev. Lett., 2014, 112, 238102-.

4 V. M. Slepukhin, M. J. Grill, Q. Hu, E. L. Botvinick, W. A. Wall and A. J. Levine, Proceedings of the National Academy of Sciences, 2021, 118, year.

5 V. M. Slepukhin and A. J. Levine, arXiv preprint arXiv:2103.08832, 2021.

6 H. A. Kramers, Physica, 1940, 7, 284-304.

7 F. C. MacKintosh, J. Käs and P. A. Janmey, Phys. Rev. Lett., 1995, 75, 4425-4428.

8 D. Strehle, P. Mollenkopf, M. Glaser, T. Golde, C. Schuldt, J. A. KÃd's and J. SchnauÃß̧, Molecules, 2017, 22, year. 